\title{
Reduction of types of Noises in dental Images
}

\author{
P.Subbuthai \\ Department of Electronics and \\ Instrumentation \\ Bharathiar University \\ Coimbatore \\ India
}

\author{
K.Kavithabharathi \\ Department of Electronics and \\ Instrumentation \\ Bharathiar University \\ Coimbatore \\ India
}

\author{
S.Muruganand \\ Department of Electronics and \\ Instrumentation \\ Bharathiar University \\ Coimbatore \\ India
}

\begin{abstract}
This paper presents a filter for restoration of Dental images that are highly corrupted by salt and pepper noise and speckle noise, Poisson noise. After detecting and correcting the noisy pixel, the proposed filter is able to suppress noise level. In this paper for each noise proposed different type of filter and compare these three types of filter with their PSNR value and MSE value and SNR value. After filtering stage maximum detected noise pixels will be filtered and simulation results show the filtered image.
\end{abstract}

Index Terms - Dental Image, FIR Filter, Gaussian Filter, Median Filter, Poisson Noise, Salt and Pepper Noise, Speckle Noise.

\section{INTRODUCTION}

The medical imaging devices particularly X-ray, CT/MRI and ultrasound are manufacturing overabundant pictures that are utilized by medical practitioners within the method of designation. The most downside visage by them is that the noise introduced attributable to the consequence of the coherent nature of the wave transmitted. These noises corrupt the image and sometimes cause incorrect designation. Every of those medical imaging devices are littered with differing types of noise. As an example, the x-ray pictures are typically corrupted by Poisson noise, salt and pepper noise, speckle noise.

The requirement to get rid of salt-and-pepper noise is very necessary before succeeding image process tasks are disbursed as a result of the contamination of image by salt-and-pepper noise is caused in great deal and therefore the occurrence of noise will severely harm the knowledge or data contained within the original image. The only and therefore the traditional thanks to take away salt-and-pepper noise is by windowing the noisy image with a standard median filter [1].

Spatial filtering is that the commonest technique for speckle noise reduction. The averaging technique is that the most well-liked linear filtering methodology among the varied spatial filters that with success removes noise from a distorted image however it's the impact of probably blurring the image.[2],[3],[4].

In [5] is conferred FIR filter based mostly Genetic mixed noise removal. A window of size $5 * 5$ is taken into account. If the window is found in no abrupt changes in grey levels i.e. flat area, estimation of central pixel is that the average of all the constituents in window closes the central constituent. If it's not flat space, abrupt changes in intensity of the constituent, estimation of central constituent is that the averages of solely similar constituent close the central pixels. So as to avoid conversion between real valued and bit string, real valued chromosomes square measure used instead of bit strings.
In [6] bestowed spatially adjustive denoising algorithmic program for one image corrupted by Gaussian noise. During this work, local statistics like local weighted mean, local weighted activity and native maxima are accustomed observe the noise. So as to suppress the additive noise, a spatially additive Gaussian filter is employed. as a result of this filter is associate adequate thanks to handle the degree of local smoothness since it's pictured as perform of native statistics. During this planned methodology, the parameters like process price, over-smoothness, detection error, smoothing degree of re-constructed image ar taken in to associate account to effectively take away the noise elements.

\section{TYPES OF NOISE}

\subsection{Salt and Pepper Noise}

A typical variety of impulse noise in a very medical image is salt and pepper noise that represents itself as every which way occurring white (salt) and black (pepper) pixels. The noise density could be a term accustomed quantifies the number of salt and pepper noise in a picture. A complete noise density of nd in an $\mathrm{M} \times \mathrm{N}$ image means $\mathrm{nd} \times \mathrm{M} \times \mathrm{N}$ pixels contain noise. In general, if the whole noise density of a salt and pepper noise is $\mathrm{d}$, then it implies that every of the salt noise and therefore the pepper noise includes a noise density of nd/2. It's attainable that the salt noise and therefore the pepper noise have completely different noise densities as nd1 and nd2, and consequently the whole noise density is nd $=$ nd $1+$ nd 2

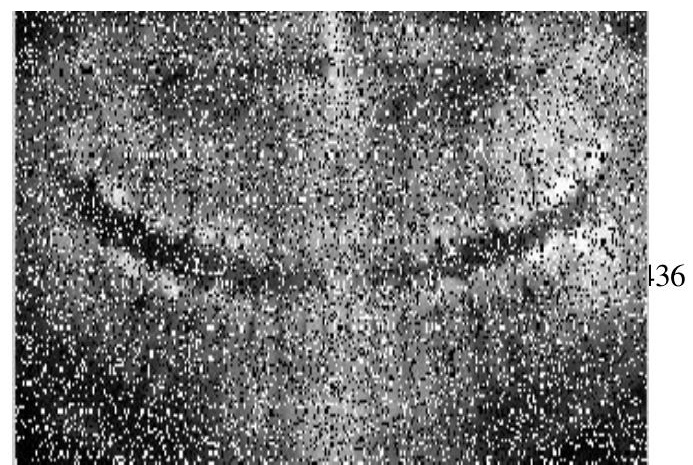


Fig.1. Salt and Pepper Noise

\subsection{Speckle Noise}

The speckle noise model may be approximated as multiplicative and is given by

$n_{i, j}=n f_{i, j} m_{i, j}+a_{i, j}$

Where $n_{i, j}$ represent the noisy pixel and $\mathrm{nf}_{\mathrm{i}, \mathrm{j}}$ represent the noisy free pixel, $m_{i, j}$ signify the multiplicative noise and $a_{i, j}$ indicate the additive noise respectively $i, j$ are indices of the spatial locations. Because the effect of additive noise is considerably smaller compared with that of multiplicative noise (1) may be written as

$$
n_{i, j}=n f_{i, j} m_{i, j}
$$

Logarithmic compression is applied to the envelope detected echo signal in order to fit within the display range [7]. Logarithmic compression affects the speckle noise statistics and it becomes very close to white Gaussian noise. The logarithmic compression transforms multiplicative form in (2) to additive noise form as

$$
\begin{aligned}
& \log \left(n_{i, j}\right)=\log \left(n f_{i, j}\right)+\log \left(m_{i, j}\right) \\
& x_{i, j}=y_{i, j}+n_{i, j}
\end{aligned}
$$

The term $\log \left(n_{i, j}\right)$ is the noisy image in the medical image after logarithmic compression is denoted as $x_{i, j}$ and the term $\log \mathrm{nf}_{\mathrm{i}, \mathrm{j}}, \log \mathrm{m}_{\mathrm{i}, \mathrm{j}}$ these are the noise free pixel and noisy component after logarithmic compression, as $\mathrm{y}_{\mathrm{i}, \mathrm{j}}, \mathrm{n}_{\mathrm{i}, \mathrm{j}}$ respectively

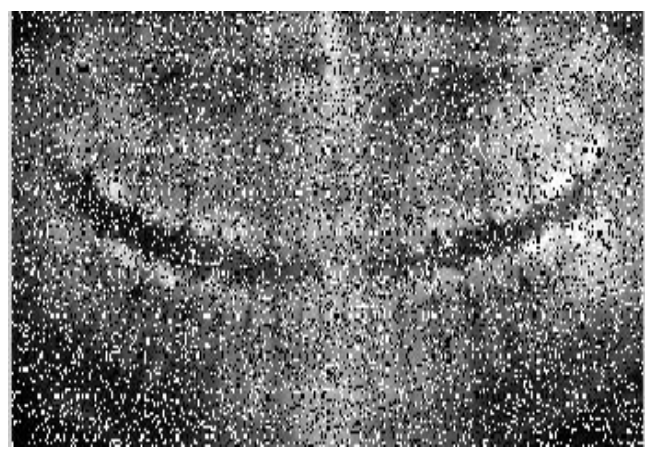

Fig.2. Speckle Noise

\subsection{Poisson Noise}

The noise in X-ray imaging and Nuclear Imaging (PET, SPECT) is sculptural with Poisson noise. X-ray photons incident on a receptor surface in an exceedingly random pattern. We have a tendency to can't force them to be evenly distributed over the receptor surface. One space of the receptor surface might receive a lot of photons than another space, even once each the area are exposed to identical average $\mathrm{x}$-ray intensity. Altogether medical imaging procedures victimization gamma or $\mathrm{x}$-ray photons most of the image noise is made by the random behavior of the photons that area unit distributed at intervals the image. This is usually selected quantum noise. Every individual photon could be a quantum (specific quantity) of energy. It's the quantum structure of associate x-ray beam that makes quantum noise [8].

A Poisson noise assume that each pixel $x$ of an image $f(x)$ is drawn from a Poisson distribution of parameter $\lambda=f_{0}(x)$ where $f_{0}$ is the original image to recover. The Poisson density is given as

$$
\mathrm{p}(\mathrm{f}(\mathrm{x})=\mathrm{k}) \text { Is equal to } \lambda^{\mathrm{k}} \mathrm{e}^{-\lambda} / \mathrm{k} \text { ! }
$$

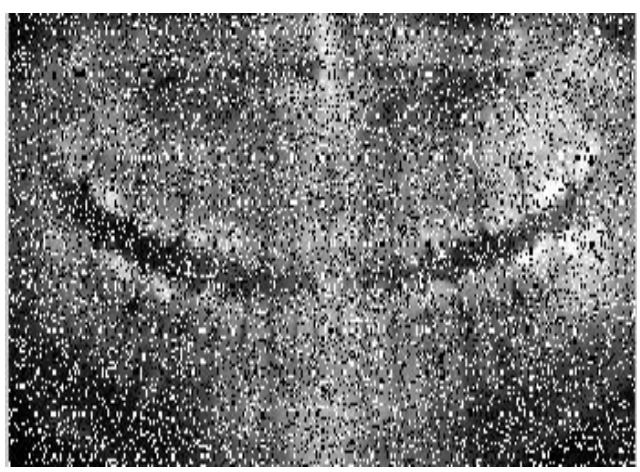

Fig. 3. Poisson Noise

\section{PROPOSED METHODOLOGY}

The quality of image is corrupted by various noises in its acquisition and transmission as shown in fig 1, 2, 3. Image denoising becomes a major problem in the field of image processing [9]. Several noise reduction techniques are available for removing noise. Various algorithms are used to denoise the noisy image and individual filtering process is used to reduce the noise level. Due to the loss of edges the image is either blurred or over smoothed. Noise reduction is used to remove the noise without losing detail present in the images.

In existing algorithms such as SMF and AMF, median values are used to replace the corrupted pixels. But it fails in case of high noise density. To overcome this drawback, proposed method uses Iterative Decision Based Algorithm. At first, the proposed algorithm extracts the pixels corrupted by using three cases of windows such as minimum, maximum and median. By checking the pixel element value against the maximum and minimum values in the window selected, the corrupted and uncorrupted pixels in the image are detected.

The maximum and minimum values of the impulse noise will be in the dynamic range of $(0,255)$. If the currently processed pixel has a value within the minimum and maximum values in the processing window, it is an uncorrupted pixel and it does not requires any modification. If the value is not within the range, the pixel becomes corrupted pixel and it will be replaced by either the median pixel value or by the mean of the neighborhood processed pixels (if the median itself is noisy).It results in smooth transition among the pixels. In the case of high noise density, the median value should be noisy. Therefore, the pixel value is replaced by the mean of the neighborhood processed pixels. 


$$
\left[\begin{array}{ccc}
\mathrm{P}_{1} & \mathrm{P}_{2} & \mathrm{P}_{3} \\
\mathrm{P}_{4} & \mathrm{C} & \mathrm{Q}_{1} \\
\mathrm{Q}_{2} & \mathrm{Q}_{3} & \mathrm{Q}_{4}
\end{array}\right]
$$

In the $3 \times 3$ window, $\mathrm{P} 1, \mathrm{P} 2, \mathrm{P} 3$ and $\mathrm{P} 4$ indicates the pixel values which are processed already, $\mathrm{C}$ indicates the current pixel to be processed, and Q1, Q2, Q3 and Q4 indicates the pixels which are yet to be processed. If the median value of the window becomes noisy, then the current pixel value $\mathrm{C}$ will be replaced by the mean of the neighborhood processed pixels P1, P2, P3 and P4. The values of the pixels Q1, Q2, Q3 and Q4 should not be considered since they indicate the unprocessed pixels.

The steps of the algorithm are elucidated as follows:

- $\quad$ Select a two dimensional window $\mathrm{W}$ of size $3 \times 3$. Assume that the pixel being processed is $\mathrm{Cx}, \mathrm{y}$.

- Compute $-\mathrm{W}$ min, $\mathrm{W}$ med and $\mathrm{W}$ max - the minimum, median and maximum of the pixel values in the window $\mathrm{W}$ respectively.

- Case a: If $\mathrm{W} \min <\mathrm{Cx}, \mathrm{y}<\mathrm{Wmax}$, then $\mathrm{Cx}, \mathrm{y}$ is an uncorrupted pixel and its value is left unchanged. Otherwise $\mathrm{Cx}, \mathrm{y}$ is a noisy pixel.

- Case b: If $\mathrm{Cx}, \mathrm{y}$ is a noisy pixel, it will be replaced by Wmed, the median value, only if $\mathrm{Wmin}<\mathrm{Wmed}<$ Wmax.

- $\quad$ Case c: If Wmin $<$ Wmed $<$ Wmax is not satisfied, Wmed itself is a noisy pixel value. In this case, $\mathrm{Cx}$, y will be replaced by the mean of the neighborhood processed pixels.

- Repeat Steps 1 to 3 until all the pixels in the entire image are processed.

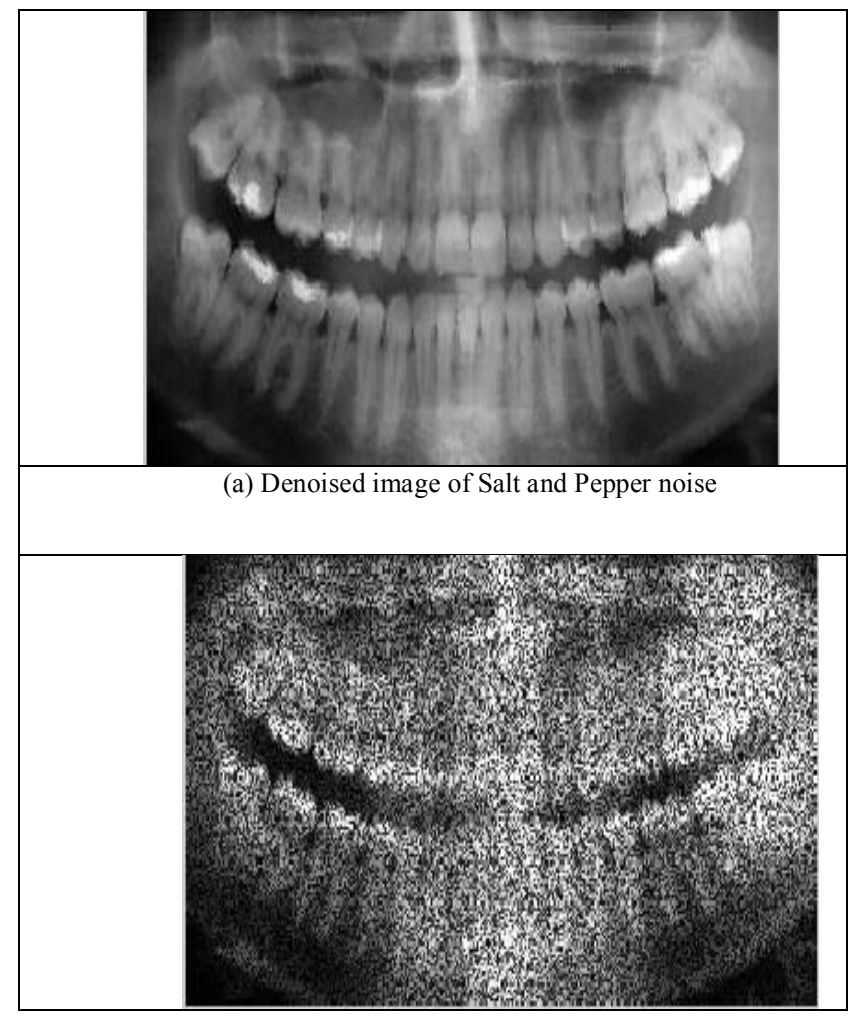

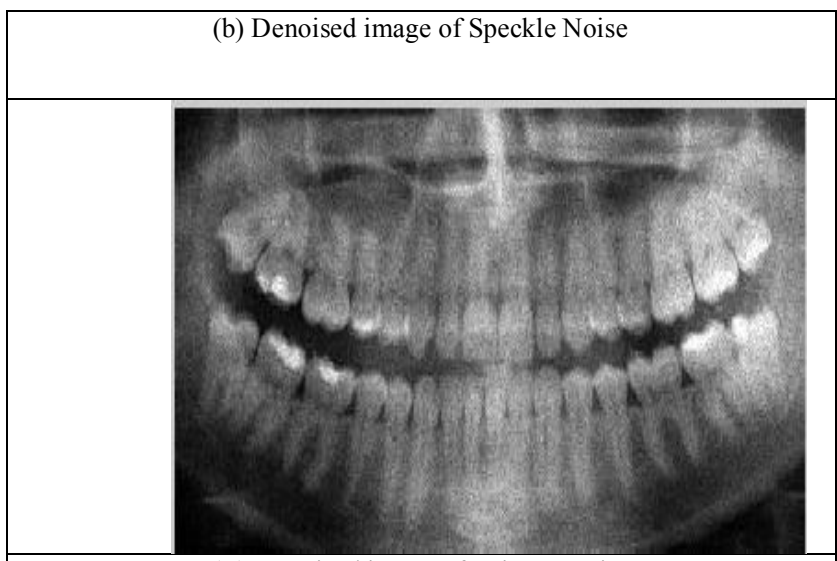

(c ) Denoised image of Poisson Noise

Fig. 4. Denoised image

In these proposed output images also have some noise. So different type of filters are used to remove the noises in these image

\section{TYPES OF FILTERS}

\subsection{Median Filter}

Filtering is a part of image enhancement it is used to enhance certain details such as edges in the image that are relevant to the application. Additionally thereto, filtering can even be used to eliminate unwanted components of noise. Medical images typically contain salt and pepper noise and Poisson noise. This noise appears owing to the presence of minute grey scale variations within the image. Median filtering could be a widespread technique of the image improvement for removing noise without effectively reducing the image sharpness [10].

Median filter is kind of common as a result of it provides excellent noise-reduction talents, with primarily less blurring than similar size linear smoothing filters. Here, the median method was performed by simply a $3 \times 3$ windowing operator over the image. It considers each pixel and its neighbors in pictures to search out whether or not it's an illustration of the environment. It replaces the value of component with the median of the neighboring pixel components. Tend to calculate the median by sorting the whole component values from the neighborhood into numeral sort then replaced the component being studied with the middle component worth. If the neighborhood below condition constitutes a good pixel worth, the common of the 2 middle component values is that the median.

\subsection{FIR Filter}

As we know, linear system parameterization is Associate in an important category of system modeling with a large space of applications. The foremost standard among the category of linear model is that the finite impulse response (FIR). It's obligatory in order to modify the estimation task and to scale back the computational load in time period application. Let $u(i, j)$ be the input of a linear 2DFIR model, defined over a regularly spaced lattice $(i, j) \in\left[I_{1}, I_{2}\right]$, where $I_{1}, I_{2}$ specify the order of the input data. The output of the 2D finite impulse response (FIR) digital filter, $y(i, j)$ is given by $2 \mathrm{D}$ finite impulse response (FIR) digital filter, $y(i, j)$, is given by 


$$
\mathrm{y} * \mathrm{i}, \mathrm{j})=\operatorname{summation}(\mathrm{w}(\mathrm{t}, \mathrm{I}) \mathrm{u}(\mathrm{i}-\mathrm{t}, \mathrm{j}-1))
$$

Where the value of $t=0$ to $N_{1}-1, I=0$ to $N_{2}-1$ and $u(i, j)$ is the input signal $w(t, I)$ is the model coefficients and $N_{1}, N_{2}$ specify the order of the FIR filter. Usually, the 2D signal is presented as a matrix. Therefore, the weight matrix $\mathrm{w}(\mathrm{i}, \mathrm{j})$ and then input matrix $(\mathrm{i}, \mathrm{j})$.

\subsection{Gaussian Filter}

Among all linear filters, Gaussian filter perhaps plays the most important role in both theory and applications. Gaussian filtering is a commonly used image filtering technique which is a WAP with weights defined as $\mathrm{w}_{\mathrm{ij}} \propto \exp \left(-\left\|\mathrm{x}_{\mathrm{i}}-\mathrm{x}_{\mathrm{j}}\right\|\right), \mathrm{i} \neq \mathrm{j}$

$$
\mathrm{w}_{\mathrm{ij}}=0
$$

Where $\|\cdot\|_{2}$ is the $\mathrm{L} 2$ norm. Because of the rapid decay of $\mathrm{w}_{\mathrm{ij}}$ as a function of distance. Gaussian smoothing is effectively a local filtering method. As an image denoising algorithm Gaussian filter is well known to over smooth images, resulting in the loss of significant detail, especially edge sharpness.

\section{EXPERIMENT RESULTS}

This section discusses the experimental results that obtained by applying the previously described median filter, fir filter, Gaussian filter to the salt and pepper noise, speckle noise, Poisson noise .

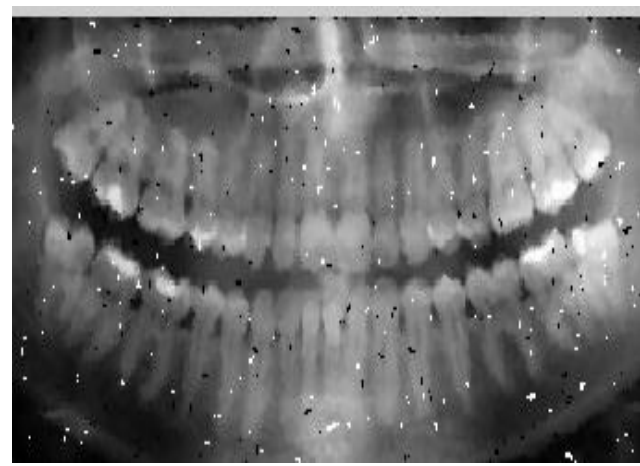

(a)Removal of salt and pepper noise using Median filter

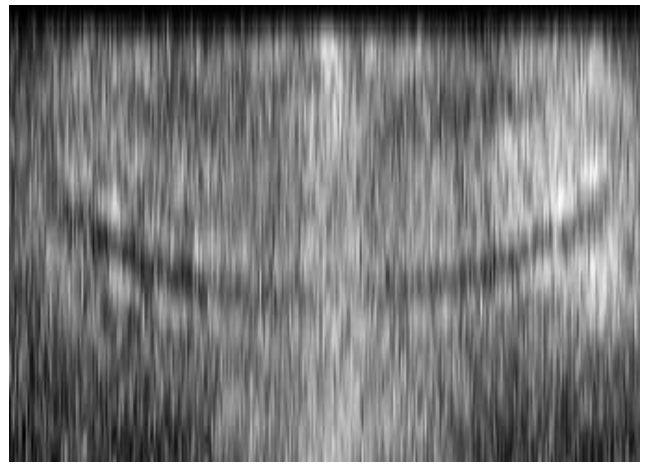

(b) Removal of salt and pepper noise using Fir filter

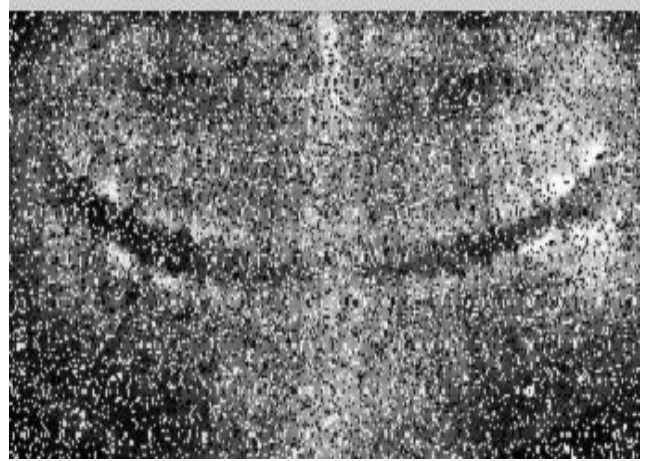

(c) Removal of salt and pepper noise using Gaussian filter

Fig. 5 removal of salt and pepper noise using different filters

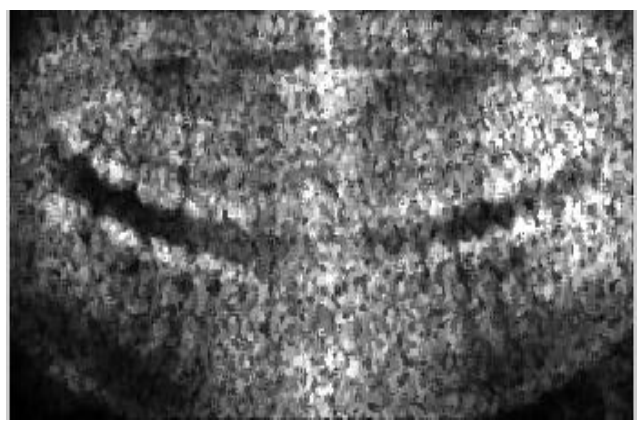

(a) Removal of speckle noise using median filter

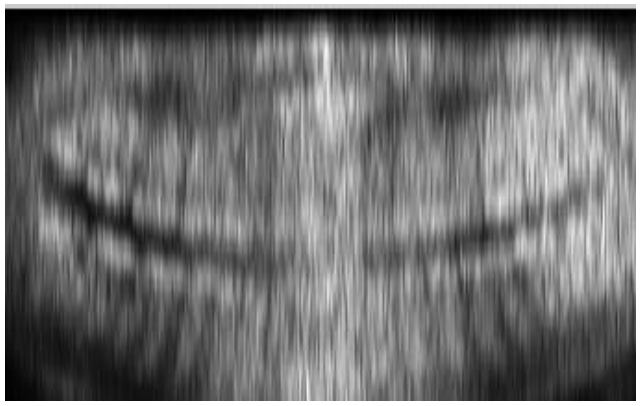

(b) Removal of speckle noise using Fir filter

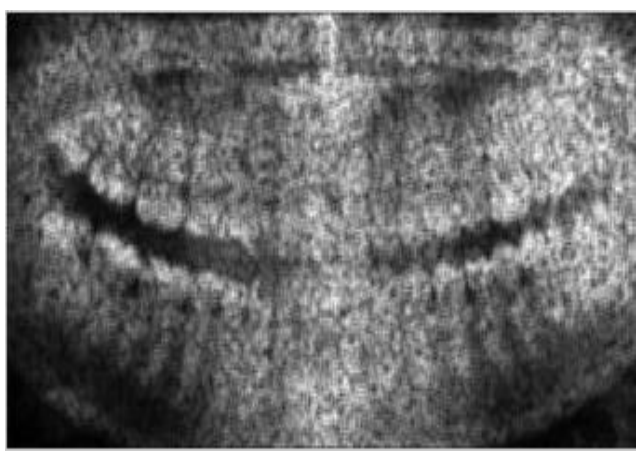

(c) Removal of speckle noise using Gaussian filter

Fig. 6 Removal of speckle noise using different filters 


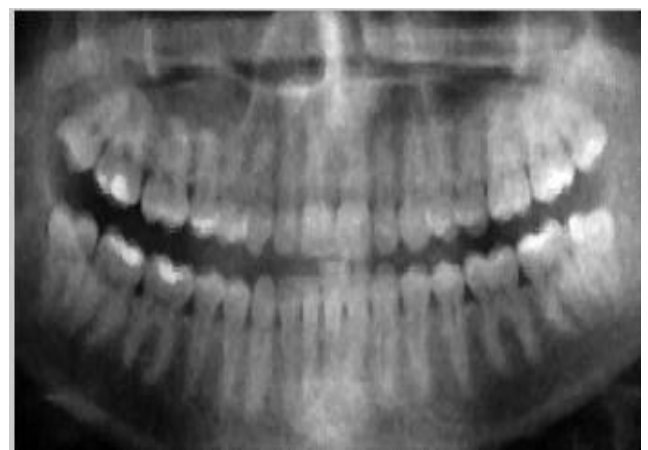

(a) Removal of Poisson noise using median filter

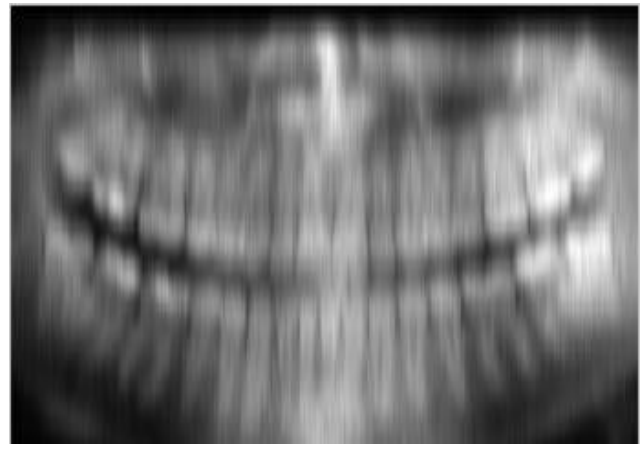

(b) Removal of Poisson noise using Fir filter

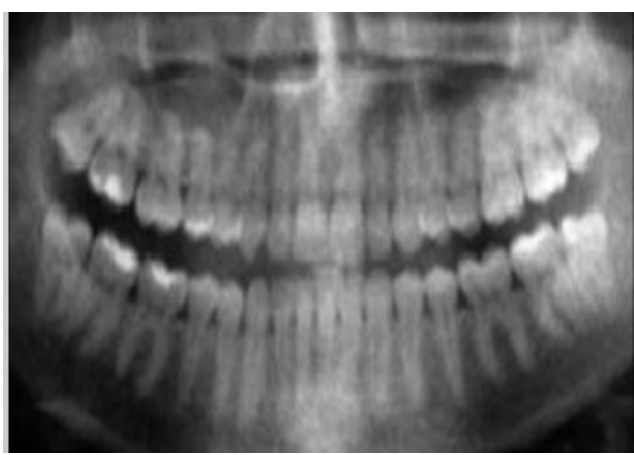

(c) Removal of Poisson noise using Gaussian filter

Fig. 7 Removal of Poisson noise using different filters

We show experimental evidence of the arguments proposed in Fig 5, 6, 7. First, our interest is in determining if the quality metrics based on the Mean Square Error (MSE) are sufficient tools in determining the quality of denoised images. For this, we measure the MSE and PSNR and SNR of denoised images.

\subsection{PSNR}

PSNR is the Peak Signal-to-Noise ratio in decibels (dB). The PSNR is only meaningful for data encoded in terms of bits per sample, or bits per pixel.

PSNR $=20 \log 10(255 / \sqrt{\mathrm{MSE}})$

\subsection{MSE}

MSE is defined as mean square error
Error $=\operatorname{abs}(A-B)$

$\operatorname{MSE}=\left(\sqrt{\left(\operatorname{mean}\left(\text { mean }\left(\text { Error. }^{\wedge} 2\right)\right)\right)}\right)$

Where Error is difference between the absolute value of $\mathrm{A}$ and $\mathrm{B}$ Where $\mathrm{A}$ is the filtered image and $\mathrm{B}$ is the Denoised image

\subsection{SNR}

SNR is defined as Signal to Noise Ratio, it is calculated as

$\mathrm{dv}=\operatorname{var}(\mathrm{B}(:), 1)$

$\mathrm{SNR}=10 * \log 10(\mathrm{dv} / \mathrm{MSE})$

Where $\mathrm{dv}$ is used to calculate the variance of the denoised image of B

TABEL 1 Salt and Pepper Noise

\begin{tabular}{|c|c|l|l|}
\hline Filter & MSE & SNR & PSNR \\
\hline $\begin{array}{c}\text { Median } \\
\text { Filter }\end{array}$ & 16.1102 & 21.5710 & 23.9888 \\
\hline FIR Filter & 39.5464 & 17.6709 & 16.1887 \\
\hline $\begin{array}{c}\text { Gaussian } \\
\text { Filter }\end{array}$ & 29.4191 & 18.9557 & 18.7582 \\
\hline \multicolumn{2}{|l}{} \\
\hline
\end{tabular}

TABEL 2 Speckle Noise

\begin{tabular}{|c|l|l|l|}
\hline Filter & MSE & SNR & PSNR \\
\hline $\begin{array}{c}\text { Median } \\
\text { Filter }\end{array}$ & 55.0061 & 19.8825 & 12.8950 \\
\hline FIR Filter & 66.5863 & 19.0528 & 11.6631 \\
\hline $\begin{array}{c}\text { Gaussian } \\
\text { Filter }\end{array}$ & 57.7815 & 19.6688 & 11.7826 \\
\hline
\end{tabular}

TABLE 3 Poisson Noise

\begin{tabular}{|c|l|l|l|}
\hline Filter & MSE & SNR & PSNR \\
\hline $\begin{array}{c}\text { Median } \\
\text { Filter }\end{array}$ & 10.3225 & 23.7178 & 27.8551 \\
\hline FIR Filter & 35.5534 & 18.3468 & 17.1132 \\
\hline $\begin{array}{c}\text { Gaussian } \\
\text { filter }\end{array}$ & 10.8411 & 23.5049 & 27.4293 \\
\hline
\end{tabular}

To evaluate the filter effectively, three main statistical measures are used. They are PSNR, MSE, and SNR. To compare these output values and find out the better filtering result of denoised X-ray images.

In table 1 the performance of various filter for salt and pepper noise. Each filter in table 1 is tested and compared with other type of filter by using of output value of PSNR, MSE, and SNR. In this median filter technique is comparati vely good for high density noise. It is observed

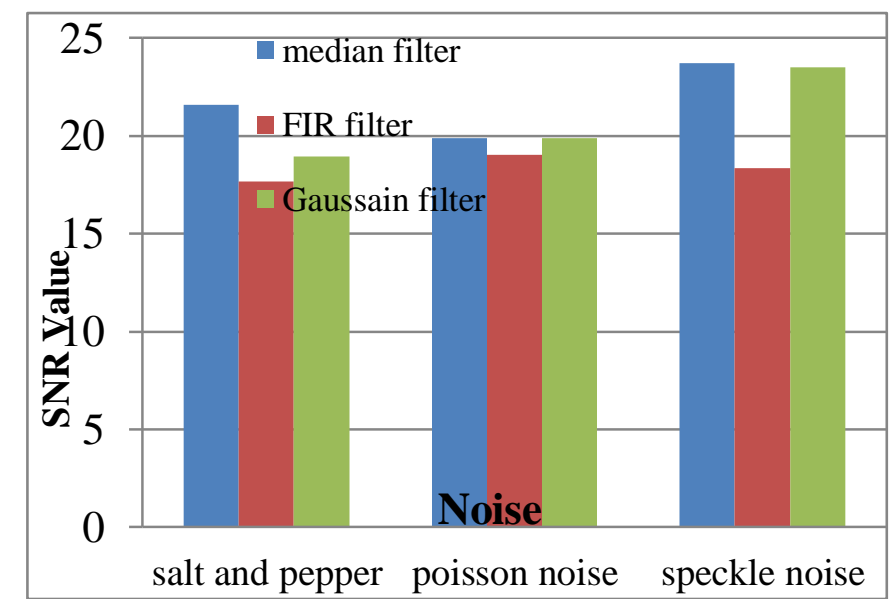


that the filter have PSNR and SNR is high compared to other filters and also it have low MSE value.

The second categories of the filtering method for the speckle noise has taken for the discussion and performed with three types of filters are shown in table 2. Median filter gives best result compared with other type of filters. In overview of all the filters in table2, median filter gives much improved results and providing a high degree of performance.

The third categories of this filtering method for the Poisson noise are shown in table 3. Here also median filter gives a high performance other than other type of filters.

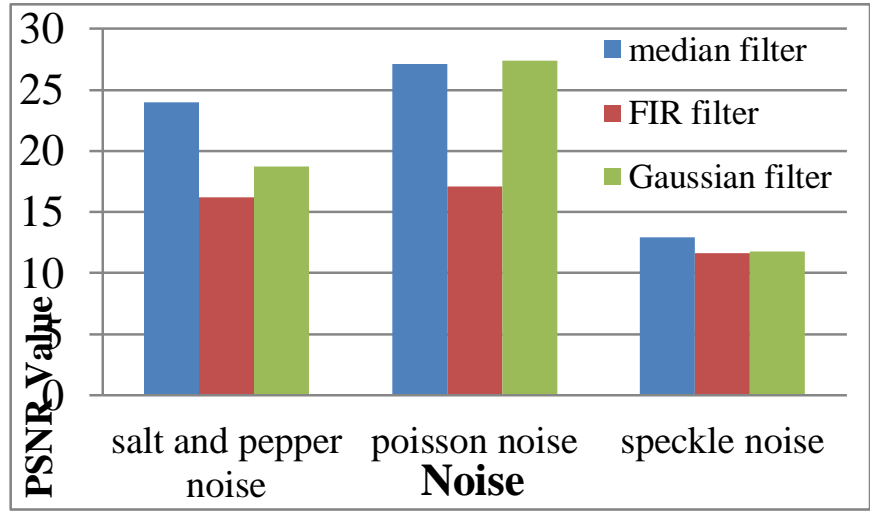

Fig. 8 Comparison of PSNR value for three type of Noise using different filters

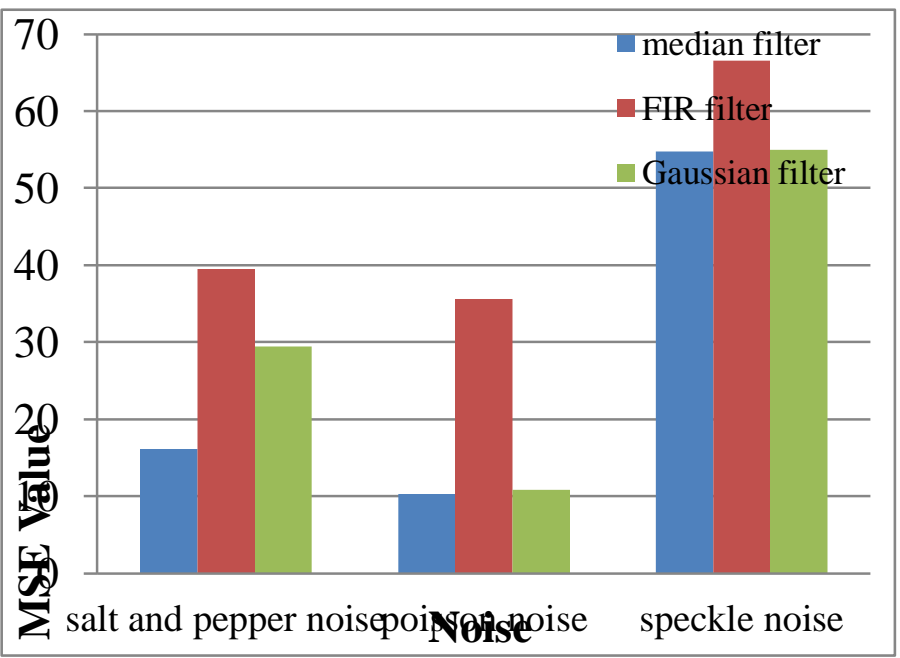

Fig. 9 Comparison of MSE value for three type of noise using different filter

Figure 8 shows the Peak Signal-to-Noise-Ratio improvement as function of the noise level for the proposed filters and, for comparison, of median filter, Fir filter, Gaussian filter. In this three type of noises are salt and pepper noise, Speckle noise, Poisson noise. In this Poisson noise have high PSNR value used by median filter.

Figure 9 shows Mean Squar Error for three type of noise used filtering of median filter, Fir filter, Gaussian filter. Comparsion of MSE value in figure 9, Poisson noise have low MSE value done by median filter.
Fig. 10 Comparison of SNR Value for three type of noise using different filter

Figure 10 shows the Signal to Noise Ratio. Here also poisson noise have hign SNR value are done by median filter.

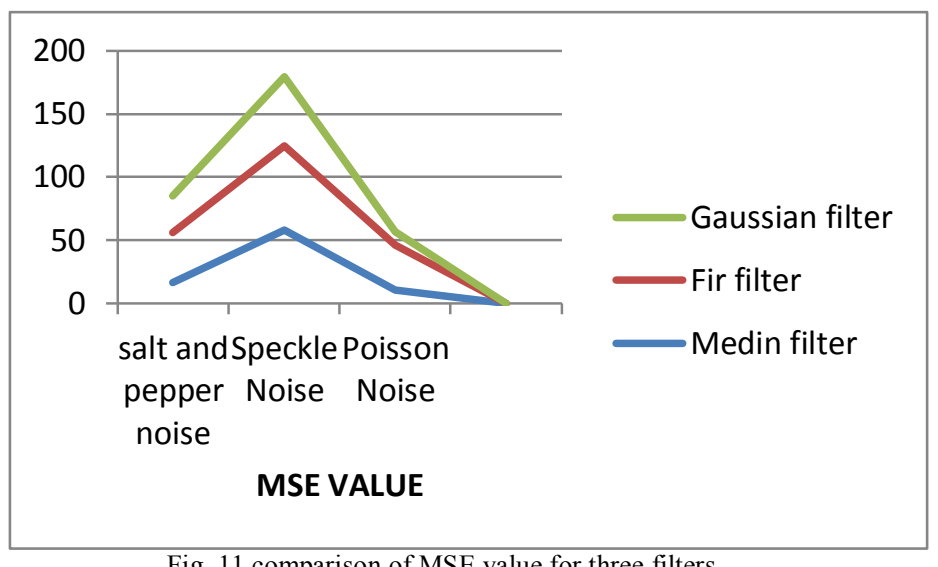

Fig. 11 comparison of MSE value for three filters

From figure 11 clearly noticed that the median filter provide better performance

\section{DISCUSSION AND CONCLUSION}

As per discussed in X-ray dental. After finding the salt and pepper noise in X-ray image various filtering techniques have been applied and it is found that the median filter works better for the noisy image. PSNR value is 23.9888 and MSE value is 16.1102

After finding the speckle noise in X-ray image various filtering techniques have been applied and it is found that the median filter works better for the noisy image. PSNR value for this 12.8950 and MSE value 55.0061 and SNR value 19.6688

After finding the Poisson Noise in X-ray image various filtering techniques have been applied and it is found that the median filter works better for the noisy image. PSNR value 27.8551 and MSE value 10.3225 and $S N R$ value 23.7178.

In this work we have taken medical images X-ray dental image for detecting noises. We have detected Salt \& Pepper noises and speckle noise and Poisson noise also removed these noises from the above medical images by applying the various filtering techniques like Median Filtering, FIR filtering, Gaussian filtering. The results are analyzed and compared with standard pattern of noises and also evaluated through the quality metrics like MSE, SNR, and PSNR. Through this work we have observed that the choice of filters for de-noising the medical images depends on the type of noise and type of filtering technique, which are used. It is remarkable that this saves the processing time. And also compare three types of filter and observe median filter gave the best accuracy. This experimental analysis will improve the accuracy of $\mathrm{X}$-ray dental image. The results, which we have achieved, are more useful and they prove to be helpful for general medical practitioners to analyze the symptoms of the patients.

\section{REFERENCES}

[1]. Kh. Manglem Singh, -Fuzzy Rule based Median Filter for Gray-scale Images, Journal of Information Hiding and Multimedia Signal Processing, Volume 2, Number 2, April 2011. 
[2]. 2. Faten A.A.Dawood, Rahmita W.Rahmat, and Suhaini B. Kadiman et al. "Automatic boundary detection of wall motion in two dimensional echocardiography images". Journal of computer science. J. computer sci., 2011, 7(8), pp: 1261-1266.

[3]. 3. Md. Robiul Hoque, and Md. Rashed-al-Mahfuz. "A new approach in spatial filtering to reduce speckle noise". International journal of soft computing and Engineering. IJSCE, 2011, volume-1, Issue-3, pp: 29-30.

[4]. 4. Juan Zapata and Ramon Ruiz. "On Speckle noise reduction in Medical Ultrasound Images", Recent advances in signals and systems, 2009, pp:126-131.

[5]. M.S. Safari, A. Aghagolzadeh," FIR filter based FuzzyGenetic Mixed noise removal", IEEE 2007.

[6]. Tuan-Anh Nguyen, Won-Seon Song, Min-Cheol Hong," Spatially Adaptive Denoising algorithm for a single image corrupted by Gaussian noise", IEEE Transaction on Consumer Electronics, Vol. 56, No. 3, Aug 2010, pp 1610-1615

[7]. V.Dutt, "Statistical analysis of ultrasound echo envelope,"Ph.D. dissertation, Mayo Graduate School, Rochester, 1995.

[8]. D.T. Kuan and A.A. Sawchuk, "Adaptive noise smoothing filter for images with signal dependent noise," IEEE Trans. Pattern Anal. Mach. Intell., vol. PAMI-7, no. 2, pp. 165-177, 1985.

[9]. Rafeal C. Gonzalez and Richard E. Woods, "Digital image processing," 2"'t, ed. Addison Wesley Longman, 1999.

[10].Chan, R.H., C.W. Ho and M. Nikolova, Salt-and pepper noise removal by median-type noise detectors and detail-preserving regularization. IEEETrans. Image Proc., 14: 1479-1485. 2005 